\title{
Pensar desde el diseñador: aproximaciones a una metodología para el relevamiento epistemológico de otros diseños
}

\begin{abstract}
Resumen
El presente artículo expone una perspectiva ontológica y metodológica que involucra las historias de vida como método y el conocimiento situado como enfoque con la intención de proponer una plataforma para el relevamiento epistemológico de "otros diseños".

En la configuración de lo que entendemos como diseño se puede reconocer algunas estructuras de tipo social en medio de las que se instituyen centros de producción de conocimiento en diseño, los cuales se han encargado de definir y agendar teórica y operativamente lo que se reconoce por este. Igualmente, de manera periférica, se desarrollan y desenvuelven otras organizaciones productoras de diseño; periferias que se comportan de acuerdo al grado de cercanía con los centros de producción institucionalizados en la definición del diseño.
\end{abstract}

Organizaciones periféricas como entornos propios de vivencia del diseño de un grupo definido de individuos, individuos con una experiencia 'otra' del diseño por su misma condición periférica. Asimismo, se reconocen condiciones que tienden a particularizar el conocimiento que desde estos 'lugares' se produce.

Desde la teoría del conocimiento situado, que propone un privilegio epistemológico de aquellos individuos que se ven sometidos a las estructuras de dominación que los marginaliza — privilegio dado por su experiencia y la capacidad de comprensión de la misma de la que solo ellos pueden dar cuenta-, se pueden conocer cosas diferentes o conocer mejor ciertas cosas frente a aquellos más favorecidos (social o políticamente).

Para hacer visible, tácito, el producto de la experiencia de estos individuos las historias de vida como método se presenta como recurso idóneo para realizar la reconstrucción de la experiencia vivida. A través de ellas es factible acceder a las relaciones entre lo subjetivo y las instituciones sociales, los imaginarios y las representaciones simbólicas, permitiendo hacer lecturas de lo social y describir el conocimiento situado sobre el diseño que se ha construido entre estos hechos de lo social.

\author{
Alejandro Rivera-Plata \\ Maestrando en Diseño \\ Investigador de la Escuela de \\ Comunicación y Bellas Artes de la \\ Corporación Unificada Nacional de \\ Educación \\ Bogotá, Colombia \\ Correo electrónico: danilo_rivera@ \\ cun.edu.co \\ azulsable@gmail.com \\ ๑ orcid.org/0000-0001-6581-0010 \\ Google Scholar
}

Recibido: Diciembre 13 de 2017

Aprobado: Septiembre 18 de 2018

Palabras clave:

Diseñador, diseño, investigación metodología, otros diseños. 


\section{Thinking from the designer: approaches to a methodology for the epistemological replacement of other designs}

\begin{abstract}
This article exposes an ontological and methodological perspective that involves life stories as a method and situated knowledge as an approach, with the intention of proposing a platform for the epistemological replacement of "other designs".

In the configuration of what is understood as design, some structures of the social type can be recognized, in the middle of which centers of production of knowledge in design are instituted that have been in charge of defining and arranging theoretically and operationally what is recognized as design. Also, peripherally, other organizations that produce design develop and unfold. These peripheries behave according to the degree of closeness with the centers of production institutionalized in the definition of the design
\end{abstract}

Peripheral organizations as environments for living the design of a defined group of individuals, individuals with a 'different' experience of design due to their peripheral condition. Likewise, conditions are recognized that tend to particularize the knowledge that is produced from these 'places'.

From the theory of situated knowledge, which proposes an epistemological privilege of those individuals who are subjected to the structures of domination that marginalize them, privilege given by their experience and the ability to understand it that only they can account for, different things can be known or certain things can be known better in front of those more favored (socially or politically).

In order to make visible and tacit the product of the experience of these individuals, life stories, as a method, are presented as an ideal resource for the reconstruction of experience lived. Through them it is possible to access the relationships between the subjective and the social institutions, the imaginaries and the symbolic representations, allowing readings of the social and describing the knowledge placed on the design that has been built between these facts of the social
Key words:

Designer, design, research, methodology, others designs. 


\section{Conocimiento en diseño}

El diseño es un atributo humano básico para ayudar a la autorrealización autónoma. Los diseñadores y los educadores para el diseño se dedican a extirpar este atributo de todas las personas, a excepción de algunos grupos cuidadosamente seleccionados, mitificando quiénes somos y que hacemos. (Papanek, 2001, p. 54)

Comprender el diseño y lo que implica el diseñar se ha formulado como obsesión para las comunidades de investigadores en diseño alrededor del mundo, lo que resulta paradójico cuando tratamos de definir como conjunto aquello que entendemos como diseño y como diseñar; pues surgen variedad de definiciones y de abordajes desde otras disciplinas que terminan por configurar una diáspora de epistemes que plantean al diseño como fenómeno de amplia complejidad.

Sin embargo, la formación de diseñadores parece restringirse a unas pocas ideas y estilos de pensamiento particulares, tendiendo entonces a desconocer la amplia complejidad que se presenta como propia del diseño.

Han sido variados los abordajes desde los cuales se ha pretendido contribuir al entendimiento y construcción de una definición del diseño; entre estos abordajes se halla el de comprender al diseño como una manera de conocimiento y al diseñar como una manera de codificar dicho conocimiento.

Entendiendo el conocimiento como un producto social, según Max Scheler, existe una forma de conocer que está vinculada a un interés propio por dominar el mundo que nos rodea; un conocimiento que se basa en lo concebido como real y en la experiencia con dicha realidad. Este conocimiento posee un valor práctico a razón de que es por medio de ese conocimiento, y de las técnicas que este deviene, que el hombre actúa sobre la realidad para dominarla (Sánchez-Migallón, 2007); es así como el conocer estaría motivado por el paso de la idea al acto. 
Sobre el acto consecuente con el conocimiento, el mismo Scheler establece que dicho acto está determinado socialmente. En el sentido de que las ideas y lo que se piensa está determinado por un sistema social, inclusive los procesos mentales por medio de los cuales el conocimiento es adquirido serian siempre determinados por las estructuras sociales; es así que aquello que conocemos lo conocemos porque existe un acuerdo social que lo define como 'meritorio' de ser conocido, ese merito se obtendría igualmente de un acuerdo social que define nuestro interés de actuar sobre esa porción de realidad, y los procesos mediante los cuales conocemos serían los procesos que en común acuerdo hemos construido como los 'correctos' para desarrollar este conocimiento.

De esta manera lo que conocemos del diseño está sometido a una selección ejecutada por una suerte de sistema social del diseño, sistema que hace las veces de estructura que se encarga de darle un rol al conocimiento adquirido y que igualmente se encarga de establecer las motivaciones que nos llevan a adquirir conocimiento en diseño y los procesos específicos que se 'deben' llevar a cabo para obtener este conocimiento en diseño.

Esta situación presenta entonces un campo de ejercicio de fuerzas y de conflicto entre el agendamiento dado por el sistema social del diseño para la construcción de una epistemología del diseño y lo que de manera particular emerge como intereses en la misma construcción de epistemes para el diseño.

Si tenemos en cuenta que esta determinación de objetos y objetivos se realiza desde perspectivas y miradas particulares, lo que Ludwik Fleck (1987) definió como "estilos de pensamiento", podemos entonces pensar que conocemos del diseño solo aquello que se ha convenido por conocer.

El impacto de los estilos de pensamiento va más allá de la negociación de objetivos, que en todo caso es crucial para la configuración de comunidades. 
El mismo Fleck (1987) describe que conocimiento científico, aquello que es conocido, es también el fruto de la homogenización que implica el establecimiento de un "estilo de pensamiento"; así, los hechos que construyen el conocimiento en diseño no son hechos dados sino que son construcciones de realidad que se hacen en función de los estilos de pensamiento predominantes. De esta manera los estilos de pensamiento son activadores para desarrollar conocimiento sobre ciertos aspectos del diseño, desarrollando instrumentos para lograr dicho conocimiento; pero a la vez inhibe de la capacidad de mirar otros aspectos y construye una visión focalizada que elude todo aquello que escapa de la estructura de realidad que concibe para sustentarse.

En este sentido la investigación en diseño ha adquirido robustez en aspectos como el proceso de diseño, sobre el que se han desarrollado una gran cantidad de estudios que en principio pretendían dar cuenta de un proceso esencial para diseñar, este es el caso de los estudios en métodos de los años 60 entre los que se destacan: la caja negra de Christopher Jones (1963), una anomalía que no se podía describir según el propio Jones; o las fases descritas por Bruce Archer (1968), que resultaron tantas que en vez de despejar la comprensión de los procesos de diseño la complejizó. Además de los enfoques sobre el problema y la manera como se despeja abordados por Alexander (1971) y Bürdek (1976) en los años 70; pasando por los estudios sobre la configuración del diseño, su materialización, de Munari (1983) y Cross (2001); y las teorías que sobre el entorno elaboraron Maldonado (1993) y Dorfles (1977); estudios y reflexiones que han dado línea para múltiples investigaciones que en los últimos 30 años han buscado dar luces sobre qué es diseñar y que últimamente han enfocado su acción en como diferenciar el diseñar de otras actividades humanas.

Esta robustez, sin embargo, ha venido eludiendo sistemáticamente algunas cuestiones que no está en capacidad de solucionar; y que en su discurso fluyen como 'curiosidades' con poca relevancia como para ubicarlas a manera de 
objeto de sus estudios. Asuntos como la definición popular de diseño o las expresiones de diseño que surgen desde individuos sin algún entrenamiento en diseñar o conceptos como "diseños otros" (Gutiérrez, 2015) o "diseño del sur" (Gutiérrez, 2014), emergen como categorías que sobre el diseño van narrando otras dimensiones que han sido ignoradas por las comunidades académicas de las instituciones que tradicionalmente han venido agendando una definición del diseño.

Entendiendo que los estilos de pensamiento han desarrollado a lo largo de los años una estructura conceptual e instrumental que resulta difícil de reconstruir para permitir espacios a otros conceptos, visiones y/u otros fenómenos, es necesario hacer un relevo en el estilo de pensamiento dominante para poder. Kees Dorst (2008), al respecto, después de realizar una anatomía de lo que ha sido la investigación en diseño en los últimos 50 años, describe en "Design research: A revolution-waiting-to-happen" la posibilidad de variar el objeto de estudio proponiendo al diseñador como centro de la investigación en diseño; si bien la manera como lo propone Dorst puede resultar superficial, al resumir al diseñador como el contenedor de capacidades para diseñar, resulta importante reconocer que en el centro de la acción del diseño se hallan individuos que han construido sus potencialidades a lo largo de una historia de vida que resulta particular.

Entendiendo que el campo de la investigación en diseño resulta ser un campo caracterizado por la pluralidad y la complejidad (Buchanan, 2007) es entonces factible iniciar abordajes desde y sobre cualquiera de los actores que se reconocen presentes en dicho campo, si se ha abordado al usuario como actor desde los enfoques del diseño centrado en el usuario (Pejtersen, 1984; Norman, 1986) es factible plantear al diseñador como objeto de estudio para un nuevo enfoque: el diseño desde el diseñador. En resonancia, con Per Galle (2008), "en el contexto social de la comunidad de investigación de diseño [...] 
opiniones diferentes estimula la discusión, por lo tanto, buenas ideas, y en última instancia, buenas investigaciones" (p. 2).

\section{Centros y periferias}

La situación planteada por los diseñadores legos actuando en la periferia de las prácticas de diseño y el tipo o los tipos de diseño que son producto de dichas prácticas, dan lugar a la configuración de un espacio donde se pueden reconocer ciertas dinámicas y tensiones que pueden considerarse propias de los fenómenos inscritos en el acto de desmarcarse de lo oficialmente prescrito, de las prácticas doctas del diseño en este caso.

La configuración de un territorio periférico del diseño desde las prácticas de los legos implica que existe un lugar que funge como centro; en principio esta idea nos permite definir la noción de periferia, dicha noción nos localiza en un lugar 'otro' que diverge del lugar construido por la academia y que diverge de los centros de producción doctos.

La academia ha sido tradicionalmente reconocida como el lugar donde el diseño tiene su origen, en este sentido Verónica Devalle (2015) sentencia que esta circunstancia plantea una paradoja para el diseño latinoamericano al haberse "constituido (los diseños) como disciplinas universitarias fuera de un esquema de industrialización [...] requisito sine qua non para algunos referentes indiscutibles del campo" (p. 26).

Esta condición de desarraigo de la industria que dio origen al diseño en otras latitudes da lugar a cuestionar algunos de los agendamientos que desde los centros académicos se distribuyen a la comunidad de diseño local. En este sentido la categoría diseñador-no diseñador, como categoría creada para definir el grado de proximidad del individuo creador respecto de los centros de producción de 
conocimiento y el grado de incorporación de los discursos hegemónicos de estos mismos centros, resulta ser una de las tecnologías del poder que implica un proceso de "selección de la realidad social" (Marcos Arévalo, 2004, p. 927) y de los individuos y termina siendo un determinante de aquello que la comunidad de diseñadores reconocemos y definimos como diseño.

Sin embargo, existe una plétora de creaciones que tienen en su origen a diseñadores formados, pero que escapan de las definiciones y categorías construidas por los propios diseñadores como expresiones de la cultura material que se ubican fuera de los territorios de estas instituciones.

\section{Las instituciones}

Las instituciones se caracterizan por ser construcciones sociales en las que el ser humano se desenvuelve, son el producto de procesos de habituación que han conducido a su institucionalización; en este sentido constituyen la trama social, la cotidianidad entendida como el cúmulo de rituales, comportamientos y emociones que rodean al ser humano en su día a día y que regula sus comportamientos al coaccionar a los individuos a seguir sus políticas mediante el ejercicio del poder que estos mismos le han otorgado.

Poseen las instituciones una trazabilidad histórica en la que se pueden reconocer ciertos mecanismos, por ejemplo, la cultura, que sirven como instrumentos para su proyección a futuro al garantizar la reproducción de las prácticas que la constituyen; definiéndose así, como parte de la herencia.

De esta manera las instituciones no solo establecen una visión hegemónica del mundo, sino que agendan una serie de actitudes y conocimientos que tienen como función prolongarse en el tiempo hasta volverse casi ineludibles para referirse a ciertas formas de la realidad. Es evidente que en el caso del 
diseño gráfico es difícil de evitar la referencia a la Escuela de la Bauhaus o a la de Ulm, por mencionar dos instituciones, a la hora de hablar de la cultura material desarrollada por el diseño mismo a lo largo del tiempo.

En las estructuras internas de las instituciones que han tributado a la definición del diseño se pueden reconocer que existen distintos roles que, según Foucault (2006), responden a la idea de separación de aquello que es correcto de lo que no lo es y que de acuerdo a Bourdieu (1987) corresponden a una estructura organizadora o 'habitus' que se encarga entre otras cosas de ubicar al individuo en el grupo; esas divisiones pueden ser atribuidas a ejercicios de poder, circunstancia que da origen a la idea de una organización que entonces se distribuye en torno al ejercicio del poder.

\section{Periferias}

Al interior entonces de estas estructuras es posible distinguir unas élites que ostentan el poder $y$, por ende, que agendan los principios con los que se reconocen las elaboraciones producto de la misma institución; a dichos principios los Ilamamos teoremas o epistemes, estos hacen parte del conjunto de tecnologías con los cuales las instituciones y los grupos de poder que las manejan aseguran su proyección a futuro.

Alrededor de estas esferas de poder se encuentran otros circuitos que poseen igualmente sus estructuras particulares y sus 'habitus' específicos: en primera instancia están los que son oficiados por los centros de poder, quienes se enrolan como seguidores, son ellos quienes ejercen la tradición y apropian las tecnologías desarrolladas por los centros de producción; luego están aquellos que se apartan de la tradición y actúan de manera divergente a las tradiciones, en términos de Foucault: ejercen la resistencia; ellos se basan en heurísticas, experiencias y la experimentación para su reflexión y acción. 
Se distinguen tres procesos que relacionan los circuitos de élites, seguidores y divergentes: el proceso de desnaturalización (Adorno, 2008) mediante el cual se induce al individuo a adoptar los principios de la tradición, como ejemplo de este proceso está la educación; el segundo proceso es el de separación o divergencia en el que los individuos cuestionan los agendamientos provenientes de las élites y anteponen a estos otras visiones y formas de ser en el mundo; el tercer proceso es el de sedimentación (Berger y Luckmann, 2003) o de legitimación donde las prácticas divergentes son adoptadas como parte de la tradición, garantizando la actualización de la tradición y asegurando su proyección a futuro.

Es así como en el diseño gráfico son reconocibles ciertos atributos del producto gráfico tales como contrastes, pesos y ritmos visuales, proporciones y unidad gráfica; atributos que se definen en las instituciones, que se conciben desde una óptica particular de las instituciones, y es singular a todos sus miembros; de manera tal que los diseños producidos bajo estos principios terminan poseyendo una cierta homogeneidad de 'escuela', como pasó en buena parte de Europa para los años 50 y 60 con los productos de la escuela suiza de la nueva tipografía. Estas escuelas de pensamiento se proyectan hasta hoy en día, expresadas en los pensum y currículos de varias academias de diseño, prolongando a través de la tradición sus postulados y nociones y así la particular materialidad de un diseño academicista y docto.

Desde esta descripción es posible reconocer como un grupo de instituciones han impuesto unas formas de ser y de circular del diseño en la cotidianidad; ahora bien, comprendiendo que estas instituciones se encuentran en continúa interacción y que fruto de esta dinámica obtenemos lo que percibimos como nuestro día a día, se puede definir que existe una 'realidad' del diseño constituida por estas interacciones. 
Igualmente, a esta construcción social del diseño se han antepuesto otras expresiones, que se han gestado y han circulado más allá de la tradición del diseño, sobre las que cabe preguntarse: cuáles son los principios sobre los que actúa —sus epistemes-; cuáles son sus prácticas específicas sobre las que plantea su divergencia - su profesión- y de qué manera se reflejan en su producción los distintos discursos que hacen parte de su acervo ideológico —su poética一.

\section{Lo que circula en las periferias}

En la periferia del diseño existe lo que se puede denominar como culturas del diseño desde las prácticas divergentes, culturas que no dependen exclusivamente de los métodos de producción, sino que recaen en las relaciones del diseño con otros actores que hacen parte del entorno de existencia del diseño mismo.

Estas manifestaciones del diseño en la cotidianidad implican la configuración de formas del diseño que despliegan en su interacción la construcción de una noción social del diseño y al diseño como un 'constructo' de carácter cultural.

Al abordar el diseño desde la perspectiva de la construcción cultural, cultura que se desarrolla en torno a los factos y los actos de diseño, estamos en posibilidad de entender a este contexto como una forma de la cultura que se define como la cultura del diseño (Margolín y Margolín, 2012). Varios de los principios de construcción de una cultura de diseño desde el ejercicio profesional han sido descritos por la academia y en este sentido han sido objeto de abstracción y teorización. Asimismo, los fenómenos que divergen del canon académico han sido objeto de censura por los gremios profesionales (asignándoles el rótulo "eso no es diseño") o han sido invisibilizados por la academia; hecho que se evidencia en la continua satanización de estos fenómenos, definiéndolos como antagonistas del canon académico. 
La construcción de una cultura de diseño consiste entonces en la construcción de una consciencia del diseño en la cotidianidad, la de varios actores; además es la definición de la presencia del diseño en cada uno de estos ámbitos; por último, es la visibilización de las relaciones que dan forma a una realidad del diseño.

De esta manera la cultura del diseño es una creación social que lo releva como práctica social, al entender que dicha cultura es fruto del diálogo y las interacciones entre los participantes en la configuración de una presencia o una forma de manifestarse del diseño.

Entonces la cultura del diseño en la periferia se constituye en los otros territorios donde coinciden los dominios de otros diseñadores (prácticas), las producciones divergentes (circulación) y el consumo del otro diseño (valor) territorio donde estos dominios interactúan y se afectan-.

\section{Los otros diseños}

En la cotidianidad latinoamericana se encuentran varias expresiones que han venido construyendo un entorno particular 'latinoamericano'. Expresiones que se han etiquetado bajo el nombre de cultura popular y que han contenido un sinnúmero de producciones, todas ellas tienen en común la negación del atributo de ser diseñadas; negación ejercida por los doctos en diseño, por las élites que definen que es diseño. Sin embargo, en su circulación cotidiana son calificadas desde las periferias como diseño; calificación que es atribuida por los individuos del común, por lo legos en diseño.

Esta diferencia diseño-no diseño expone una división en el diseño que nos rodea, división que en principio busca desconocer unas expresiones y legitimar otras como las únicas. Boaventura de Sousa expone la idea de epistemologías 
del sur (2010) al describir un conjunto de saberes y principios del saber que se presentan como alternativa a las formas del conocimiento impuestas por Occidente y las instituciones que lo encarnan.

Estas epistemologías del sur, según la definición de de Sousa, tienen como fin: denunciar la aniquilación de saberes, epistemicidios, laborada por agentes de las instituciones en su intención de afirmarse en la realidad de los individuos; y habilitar la reconstrucción y el resurgimiento de dichos saberes al reivindicar su autenticidad y pertinencia en el contexto particular latinoamericano.

En este sentido el rótulo de no diseños, que se atribuye a las materialidades gestadas y que circulan en las periferias, responde a la intensión de suprimirlos de la concepción institucional de diseño; por tanto, es entonces necesario definirlos de otra manera que no implique el 'no'.

Con base en ello Alfredo Gutiérrez Borrero (2015) propone los diseños del sur y los diseños otros, definiendo estos como:

dos formas estratégicas para designar aquí los mismos fenómenos. Intentos de provincializar el diseño [...] occidental para que deje de ser "la norma" y se convierta en "lo normal" (uno más de muchos), aluden a la construcción de artefactos a partir de las lógicas de los resurgimientos (buen vivir, tikanga maorí, ubuntu, etcétera), desde distintos marcos interpretativos. (p. 120)

Siendo entonces diseños otros la designación que más se ajusta a estas otras formas del diseño que circulan en la periferia, sobre la definición de diseños otros el mismo Gutiérrez (2015) apunta que:

\footnotetext{
"los diseños otros" se aproximan al paradigma otro de Walter Mignolo (2014), quien advierte que las divisiones este/oeste y norte/sur no son ontológicas sino ficticias y políticas, pues revelan más las intenciones de los cartógrafos (instituciones, personas y organizaciones), que la naturaleza de lo nombrado, clasificado y mapeado [...] el paradigma otro no es de transición, sino de disrupción, los "diseños otros" saldrían de saberes característicos de los resurgimientos, aplicados por gentes que podrían reivindicar su derecho a construir sus mapas o a prescindir de éstos. (p. 120-121)
} 
Son entonces diseño otros los que circulan en nuestra cotidianidad, paralelo a los diseños doctos, haciendo parte de la materialidad atribuida al diseño desde las experiencias de los seres que interactúan con estos, construyendo desde estas experiencias unas nociones de diseño que escapan de las definiciones emanadas desde las instituciones y que inquietan -y a veces molesta- a quienes nos hemos empoderado en el diseño desde el rol de diseñadores.

\section{Pensar desde el diseñador}

El diseñador en el acto de diseñar es el agente mediante el cual el diseño adquiere su forma, es desde esta perspectiva que tradicionalmente se ha reparado en el diseñador a la hora de elaborar teorías sobre el diseño: el diseñador presente como circunstancia del diseño.

Se ha abordado al diseñador como sujeto de estudio en algunas investigaciones que buscan describir los procesos de toma de decisiones, los estudios de Rittel y Webber (1973), Buchanan (1992) y Goel y Pirolli (1992) han descrito como el diseñador se aproxima a la solución acercándola a su campo de experticia. Otros escritos como los de Dorta, Pérez y Lesage (2008), Cross (2001) y Brown (2008) se han enfocado en la ideación y las herramientas utilizadas durante esta fase del diseño. Sin embargo, es poca la importancia que se le ha dado al diseñador como individuo.

\section{El diseñador situado}

Resulta relevante entender al diseñador ubicado en un marco de referencia específico, entenderlo como individuo situado, contenido por una idea de realidad que potencia ciertas características de su diseño a la vez que limita su visión para la concepción de otras características. Esta forma de abordar al diseñador como fenómeno situado surge de entender tres fenómenos 
subsecuentes: en principio, entendiendo al individuo como sujeto en construcción siendo el resultado de varios factores entre ellos su propia historia de vida; y que esos diversos factores terminan proyectados en su sistema de intereses, sus concepciones del mundo, y sus capacidades como diseñador entre otros, por ello es lógico concebir que el diseñador es un sujeto situado en construcción.

En segunda instancia, entendiendo que el conocimiento en diseño que se expresa en la producción de diseño de cada sujeto creador- es un conocimiento que "se produce en situaciones históricas y sociales particulares, por mucho que se quiera hacer aparecer el verdadero conocimiento científico como universal, neutral y por lo tanto desprovisto de relaciones directas con determinados factores políticos, culturales y sociales" (Piazzini, 2014, p. 12); de manera que ese conocimiento también posee un carácter situado.

Por último, entendiendo que la producción de diseño no se halla aislada de las condiciones de su productor; siendo la producción, el diseño mismo, un dispositivo de representación de la estructura ideológica del diseñador en sí (Rivera-Plata, 2018).

Al abordar al diseñador como sujeto de estudio entendiéndolo como un sujeto situado —siendo la situación un marco no solo para el individuo, sino también para el conocimiento que tiene del mundo (y en este caso del diseño) y para su acción en el mundo (en este caso definida por su producción de diseño) — es factible entender algunos fenómenos que tradicionalmente han sido relegados a lo anecdótico en la investigación en diseño.

Aspectos como la capacidad particular de resolver problemas, el genio o la genialidad, la postura del diseñador, su postura política, y en general todo su aparato ideológico, han sido tratados de manera superficial al considerarse 
meramente circunstancial a los estudios de caso; aun así, resultan determinantes para la definición material del diseño.

Así, entonces, el pensar desde el diseñador es encarnar varias de las fuerzas que definen al diseño en su condición material; pensar desde él permitiría entender las razones de su particular forma de definir la materialidad del diseño; igualmente permitiría entender las particularidades de las concepciones situadas del diseño en su condición epistémica. De esta manera pensar desde el diseñador generaría un aporte significativo para la construcción en complejidad de nuestro conocimiento en diseño.

\section{Aproximaciones a una metodología para el relevamiento epistemológico de otros diseños}

Elaborar una descripción de "otros diseños" con la mirada puesta en "otros diseñadores" implica una ruptura en la tradición occidentalizada de los estudios en diseño que han insistido en ubicar al diseño desde los agendamientos de las instituciones y a la vez han relegado al diseñador a una circunstancia del diseño; o en los mejores casos a un actor que se expresa bajo el rotulo de diseñador, sin más pretensión que la de materializar al diseño.

Igualmente abordar la complejidad que rodea la cotidianidad y prácticas de algunas comunidades e individuos implica el uso de métodos e instrumentos que permitan capturar dicha complejidad de quienes en sus situaciones particulares día a día han construido maneras particulares de comunicarse e intervenir sobre el mundo que los rodea. Para tal propósito se propone abordar dicha complejidad en tres momentos: un momento descriptivo; un momento analítico y un momento reflexivo.

El planteamiento del proceso de investigación implica que de manera iterativa se derive entre el trabajo de campo y la red conceptual que en principio se 
tejió a través de referentes teóricos con el fin de ir refinando cada vez tanto la capacidad de observación de las dinámicas y tensiones presentes en el campo como las categorías fruto del análisis de dichas observaciones. Igualmente, cada retorno propone una profundización en los aspectos identificados, una amplitud de los fenómenos observados y un continuo cuestionamiento sobre los registros, análisis y reflexiones.

En este sentido se proyecta un continuo ciclo de observación, análisis y reflexión que nuevamente demanda una observación; de manera que los resultados se deberían observar como fruto de un momento, de un corte, y no como conclusiones que den cuenta de la absoluta complejidad asumida que resultara siempre inconmensurable a la luz de cada dato y cada movimiento de los actores en su tiempo y espacio.

Se dispondría del levantamiento de una serie de casos que posean como común denominador el ser individuos y/o comunidades que expresen una particular manera de manifestarse en el mundo y que configuran o configuraron en su entorno una cotidianidad específica. En esa cotidianidad se plasma una visión peculiar de mundo que está contenida por una serie de códigos, una materialidad y unas prácticas que constituyen las unidades de estudio.

Con la intención de develar parte de la complejidad inmersa en las maneras de vivir de los sujetos de estudio seleccionados en cada caso a levantar se dispondría un abordaje a dichas cotidianidades y existencias desde un punto de vista hermenéutico, entendiendo esta perspectiva como el estudio más adecuado parta aproximarse a las razones de la acción humana y considerando que tales acciones tienen una organización semántica y no lógica o causal (Heidegger, 2009).

Para el levantamiento de los registros el uso de las historias de vida como método otorga la posibilidad de capturar información de la acción y experiencia 
vital de los sujetos protagonistas de cada caso, entendiendo que a través de dichas experiencias y acciones los individuos construyen y narran sobre las estructuras sociales que los envuelven y en estas la visión de mundo que los hace particulares (Miller, 2000); visión de mundo que se propone como la principal búsqueda de una investigación que pretenda relevar a los 'otros diseños', que en potencia sería el aporte al campo del conocimiento en el diseño.

El criterio de trabajo para la recolección de estas vivencias en cada caso tiene la posibilidad de variar: por un lado Bertaux (1996) propone la construcción de la visión de mundo desde los métodos biográficos tomando registro de casi cualquier contacto con el sujeto, desde esta perspectiva minimalista Bertaux propone que en casi cualquier forma de interacción del sujeto con su entorno se expresa un fragmento de sus discursos y estructuras ideológicas desde donde se puede partir para reconstruir una porción significativa de la realidad del individuo. Por otro lado Ferrarotti (1991), quien define que las historias de vida trascienden a su condición de método para convertirse en paradigma para el abordaje de otras realidades, hace énfasis en la inmensa complejidad que se teje en la realidad de cualquier sujeto y que esa realidad posee un carácter sistémico; de manera que para relevar un aspecto de la realidad de un sujeto es imprescindible hacer evidente las relaciones que este aspecto establece con otros y en ese sentido determinar las dinámicas y conexiones que hacen evidente y accesible dicho aspecto para ser estudiado y objeto de narración.

En todo caso, para este relevamiento, se exige un seguimiento profundo a las prácticas y vivencias de los individuos específicos; tratando de abarcar en el registro la totalidad de condiciones que los rodea para dar cuenta de una cotidianidad específica. 


\section{Para pensar}

Richard Buchanan (2004), en la Conferencia Internacional de la Design Research Society celebrada en Melbourne (Australia), hacía la descripción del reto que se presentaba a la investigación en diseño y definía que:

una de las fortalezas de nuestro campo (la investigación en diseño) es que tenemos diferentes puntos de vista [...]. Lo que puedo ofrecer es una perspectiva personal del campo, enmarcada por aquello que he encontrado en el tiempo y por mis propios preceptos filosóficos y abordajes al diseño.

En este sentido el abordar la investigación en diseño con perspectivas distintas a las tradicionales es en potencia un mecanismo para profundizar en el conocimiento del diseño desde una perspectiva compleja, dando luces sobre cada uno de los actores que se reconocen en la intrincada trama que se teje cuando el diseño se manifiesta.

Es así como observar el diseño desde la particularidad de quienes diseñan propende por la comprensión del origen de varios de los aspectos que determinan al diseño tales como el estilo, los abordajes, las limitantes a la solución y la injerencia de otros actores entre otros.

De igual manera entender al diseñador como individuo posibilita el entendimiento de otros fenómenos que devienen del mismo diseñador como, por ejemplo, los esquemas de enseñanza-aprendizaje del diseño; el entender cómo impacta el aprendizaje del diseño en los estilos de resolución de problemas, que el diseñador exhibe de manera situada, permitiría reflexionar de manera más argumentada los modelos de enseñanza del diseño y pensar en modelos de pedagogía del diseño más coherentes con el contexto del diseñador.

Finalmente describir los vínculos que se forjan entre el diseñador y su contexto, y de estos con su historicidad —la de cada uno-, proporciona herramientas 
para aproximarse a la comprensión de las ideas que sobre el diseño circulan en lo cotidiano; ideas que se construyen y desarrollan por fuera de las instituciones doctas en el conocimiento del diseño y que, sin embargo, sirven para definir el diseño a los que no son doctos en diseño; definiendo a los "otros diseño" por fuera de nuestro entendimiento.

\section{Referencias}

Adorno, T. (2008). Resumen sobre la industria cultural. Crítica de la cultura y sociedad I. Madrid, España: Akal.

Alexander, C. (1971). The state of the art in design methods. Design Methods Group Newsletter, 5 (3), 1-7.

Archer, B. (1968). The structure of design processes. London, England: Royal College of Art.

Berger, T. y Luckmann, T. (2003). La construcción social de la realidad. Buenos Aires, Argentina: Amorrortu.

Bertaux, D. (1996). Historias de casos de familias como método para la investigación de la pobreza. Revista de Sociedad, Cultura y Política, 1 (1), 3-32.

Brown, T. (2008). Design thinking. Harvard Business Review, 86 (6), 84-92.

Bourdieu, P. (1987). "Habitus, code, codification". Actes de la Recherche en Sciences Sociales, 64, 40-44.

Buchanan, R. (1992). Wicked Problems in Design Thinking. Design Issues, 8 (2), 8-21.

Buchanan, R. (2004). Design as inquiry: The common, future and current ground of design. Recuperado de http://www.designresearchsociety.org/ futureground/pdf/buchanan.pdf. 
Buchanan, R. (2007). Strategies of Design Research: Productive Science and Rhetorical Inquiry. En R. Michel (Ed.), Design Research Now (pp. 55-66). New York, USA: Springer.

Bürdek, B. (1976). Introducción a la metodología del diseño. Buenos Aires, Argentina: Editorial Nuevavisión.

Cross, N. (2001). Designerly ways of knowing: Design discipline versus design science. Design Issues, 17 (3), 49-55.

de Sousa, B. (2010). Descolonizar el saber, reinventar el poder. Montevideo, Uruguay: Trilce.

Devalle, V. (2015). Autocrítica. Asumir las fortalezas y debilidades de nuestra disciplina. IF, 10, 26-27.

Dorfles, G. (1977). El diseño industrial y su estética. Barcelona, España: Ed. Labor.

Dorta, T., Pérez, E. and Lesage, A. (2008). The Ideation Gap: Hybrid tools, Design Flow and Practice. Design Studies, 29 (2), 121-141.

Dorst, K. (2008). Design research: A revolution-waiting-to-happend. Design Studies, 29, 4-11.

Ferrarotti, F. (1991). La historia y lo cotidiano. Barcelona, España: Península.

Foucault, M. (2006). Sobre la Ilustración. Madrid, España: Tecnos.

Galle, P. (2008). Candidate worldviews for design theory. Design Studies, 29 (3), 267-303.

Gutiérrez, A. (2014). Chakana o la búsqueda del diseño con otros nombres. Recuperado de http://www.academia.edu/11660099/CHAKANA_O_ LA_B\%C3\%9ASQUEDA_DEL_DISE\%C3\%91O_CON_OTROS_ NOMBRES_Espa\%C3\%B1ol_2014_. 
Gutiérrez, A. (2015). Resurgimientos: sures como diseños y diseños otros. Nómadas, 43, 113-129.

Goel, V. and Pirolli, P. (1992). The Structure of Design Problem Space. Cognitive Science, 16, 395-429.

Heidegger, M. (2009). La esencia de la verdad. Barcelona, España: Herder.

Jones, C. (1963). A Method of Systematic Design. En C. Jones and D.G. Thornley (Eds.), Conference on Design Methods (pp. 53-73). Oxford, England: Pergamon Press.

Fleck, L. (1987). La génesis y el desarrollo de un hecho científico. Madrid, España: Alianza Editorial.

Maldonado, T. (1993). El diseño industrial reconsiderado. Barcelona, España: Gustavo Gili.

Marcos Arévalo, J. (2004). La tradición, el patrimonio y la identidad. Iztapalapa: Revista de Ciencias Sociales y Humanidades, 57, 925-956.

Margolín, V. y Margolin, S. (2012). Un "modelo social" de diseño: cuestiones de práctica e investigación. Revista KEPES, 9 (8), 61-71.

Miller, R. (2000). Researching Life Stories and Family Histories. London, England: SAGE.

Munari, B. (1983). ¿Cómo nacen los objetos? Barcelona, España: Gustavo Gili.

Norman, D. (1986). User centered system Design: New perspectives on Human-Computer Interaction. Hillsdale, USA: L. Erl-baum Associates.

Papanek, V. (2001). Edugrafologia: los mitos del diseño y el diseño de los mitos. En M. Morán (Ed.), Fundamentos del diseño gráfico (pp. 50-55). Buenos Aires, Argentina: Ediciones Infinito. 
Pejtersen, A. (1984). Design of a computer-aided user-system dialogue base on an analysis of user's search Behaviours. Social Science Information Studies, 4, 167-183.

Piazzini, C.E. (2014). Conocimientos situados y pensamientos fronterizos. Geopolítica(s), 5 (1), 11-33.

Rittel, H. and Webber, M. (1973). Dilemmas in a General Theory of Planning. Policy Sciences, 4, 155-169.

Rivera-Plata, A. (2018). Diseño, identidad e ideología: el diseño como discurso. Pensamiento, Palabra y Obra, 20, 94-103.

Sánchez-Migallón, S. (2007). Max Scheler. Recuperado de http://www. philosophica.info/archivo/2007/voces/scheler/Scheler.html.

Como citar: Rivera-Plata, A. (2019). Pensar desde el diseñador: aproximaciones a una metodología para el relevamiento epistemológico de otros diseños. Revista KEPES, 16 (19), 253-275. DOI: 10.17151/ kepes.2019.16.19.10 